\title{
C023
}

\section{Estimation of Thomsen Anisotropy Parameters Using the P-wave Velocities on a Cylindrical Shale Sample}

\author{
D. Nadri* (CSIRO), J. Sarout (CSIRO), A. Bóna (Curtin University) \& D. \\ Dewhurst (CSIRO)
}

\section{SUMMARY}

In this paper we present a new approach to the estimation of Thomsen anisotropy parameters from laboratory data on cylindrical rock samples. Using tomography-style transducers array, ultrasonic P-wave ray velocities are measured on a transversely isotropic shale sample. This approach is applied to core samples cut along and normal to the bedding plane. Synthetic and actual laboratory data from an anisotropic shale specimen are used as examples. The fast simulated re-annealing method is used to search for the anisotropy parameters. 


\section{Vienna ''11}

\section{Introduction}

Because of flat-lying sedimentation of clay particles, shales often behave elastically as a transversely isotropic (TI) material. The determination of Thomsen anisotropy parameters in a TI medium usually relies on very few laboratory measurements of P-waves velocities along and normal to the bedding (symmetry) plane, together with an independent measurement at an oblique angle - often at $45^{\circ}$ - to the bedding plane. This also requires measuring at least two independent $\mathrm{S}$-wave velocities, which are inherently less accurately measured in the laboratory than P-wave velocities. Few sparse measurements result in a very high uncertainty for Thomsen parameters, in particular for $\delta$. Here, a tomography-style transducers array is used to measure $\mathrm{P}$-wave ray velocities on a cylindrical sample of a transversely isotropic shale. We developed a ray-tracing based algorithm to compute the ray velocities, assuming the homogeneity of the sample. Using these measured velocities, Thomsen anisotropy parameters are estimated using a very fast simulating re-annealing algorithm. Where appropriate, a non-linear conjugate gradient algorithm has been implemented in order to tune the best estimate. We applied the methodology to a synthetic model and an actual experimental data set obtained on a shale specimen. Two configurations are analyzed, i.e., cylindrical specimen axis perpendicular (VTI) and parallel (HTI) to the shale bedding plane.

\section{Transverse isotropy with vertical symmetry axis (VTI)}

The elastic properties of a shale specimen cored normal to the bedding plane can be approximated by a transversely isotropic system with a symmetry axis coinciding with the cylinder axis. This corresponds to a situation where the shale is subjected to an isotropic horizontal stress field. In order to compute P-wave phase velocities from the measured P-wave ray velocities we use the parametric solution of the Christoffel equation for a transversely isotropic medium with vertical symmetry axis given by Ursin and Stovas (2006),

$$
\begin{aligned}
1 / v^{2} & =1 / \alpha^{2}-p^{2}(\sigma+\delta)-\left(\gamma^{2}-1\right)\left[\sqrt{1+b p^{2} \alpha^{2}+c p^{4} \alpha^{4}}-1\right] / 2 \alpha^{2}, b=-\frac{4}{\gamma^{2}-1}(\sigma-\delta), \\
c & =4\left[2\left(\gamma^{2}-1\right) \sigma / \gamma^{2}+(\sigma+\delta)^{2}\right] /\left(\gamma^{2}-1\right)^{2}, \quad \gamma=\frac{\alpha}{\beta}, \sigma=\gamma^{2}(\varepsilon-\delta),
\end{aligned}
$$

where $v$ is the phase velocity, $p$ is the ray parameter (projection of slowness vector to the bedding plane) and $\alpha, \beta, \varepsilon$, and $\delta$ are Thomsen anisotropy parameters; the parameters $\alpha$ and $\beta$ correspond to the $\mathrm{P}$ - and $\mathrm{S}$-waves velocities propagating along the symmetry axis.

Because of the relatively small size of the transducers, the measured velocities correspond rather to ray velocities, which can be different from the associated phase velocities for propagation directions other than along the symmetry axis or within the symmetry plane. The magnitude of the ray velocity is related to that of the phase velocity by

$$
V^{2}=v^{2}+(\partial v / \partial \theta)^{2},
$$

where $V$ is the P-wave ray velocity and $\theta$ is the phase angle. The derivative $\partial v / \partial \theta$ can be expressed as a function of the ray parameter (e.g. Ursin and Stovas, 2006)

$$
\frac{\partial v}{\partial \theta}=\frac{\sqrt{1-p^{2} v^{2}} \partial v / \partial p}{v+p \partial v / \partial p},
$$

where the derivative $\partial v / \partial p$ can be computed from equation 1 .

Computing the phase velocity from the ray velocity is not straightforward. We use a ray tracing approach to compute the ray parameter; we also express the ray path projection $d$ into the isotropy plane (see Figure 1), as a function of the ray parameter and the source-receiver geometry,

$$
d=\frac{h v}{\sqrt{1-p^{2} v^{2}}}\left(p+\frac{\partial v / \partial p}{v^{3}}\right),
$$




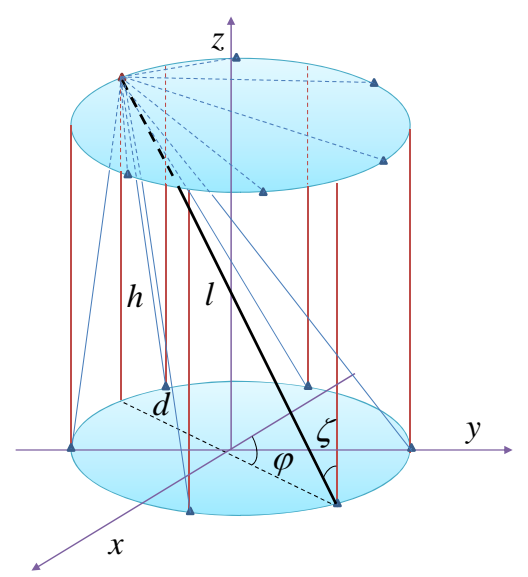

Figure 1 A schematic of ray shooting configuration for an array of source and receivers on a cylindrical shale sample. Each transducer acts as a source while the rest of the transducers act as receivers. The symbols $\zeta$ and $\varphi$ are ray and azimuth angles. The symmetry axis is along the $z$ axis. The symbol $h$ is distance between source-receivers in two isotropy planes and $l$ is the ray length. The symbol $d$ is the projection of ray path on an isotropy plane.

where $h$ is the distance between the two (isotropy) planes containing the sources and receivers (blue triangles in the figure).

We minimize the following objective function $f$ using a Newton method to solve for the ray parameter $p$,

$$
f=\frac{1}{2}(d-\hat{d})^{2}
$$

where $\hat{d}$ is the computed ray projection from equation 4 for a given ray parameter. Computing the ray parameter in an isotropy plane is straightforward, and corresponds to the slowness $1 / v$. For this case, we use the Thomsen (1986) formulation, which for rays propagating in isotropy plane simplifies to

$$
\frac{1}{p^{2}}=\alpha^{2}(1+\varepsilon+\Delta), \Delta=0.5\left(1-\beta^{2} / \alpha^{2}\right)\left[\sqrt{1+\frac{4\left(1-\beta^{2} / \alpha^{2}+\varepsilon\right) \varepsilon}{\left(1-\beta^{2} / \alpha^{2}\right)^{2}}}-1\right]
$$

\section{Transverse isotropy with horizontal symmetry axis (HTI)}

The elastic properties of a shale plug cored parallel to the bedding plane can be represented by a transversely isotropy system with horizontal symmetry axis (see Figure 2). Note that the transducers array is identical to that employed for the VTI configuration. Therefore, after computing the ray parameter $p$, equations 1 and 6 are directly used for the HTI configuration with no modification. However, in order to compute $p$, equation 4 is adapted to the HTI configuration and yields

$$
l=\frac{\sin \Delta \varphi}{\sin \zeta} \frac{d v}{\sqrt{1-p^{2} v^{2}}}\left(p+\frac{\partial v / \partial p}{v^{3}}\right), \cos \zeta=\cos \omega \sin \Delta \varphi,
$$

where $l$ is the ray length and $d$ is the projection of the ray on the $\mathrm{x}$-y plane. The angle $\Delta \varphi$ denotes the difference in azimuth between the bedding plane and the ray projection on the $x-y$ plane. The angles $\omega$ and $\zeta$ stand for the dip angle of the ray and the ray angle, respectively. Similarly to the VTI configuration, the iterative minimization of an objective function is used for computing the ray parameter from $l$. 


\section{Vienna |'11}

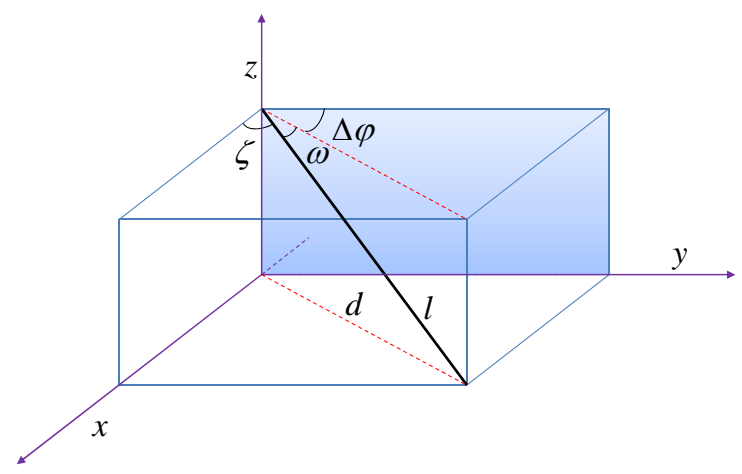

Figure 2 A schematic of a ray element in a HTI medium. The z-y plane is the isotropy (bedding) plane. The symbol $\zeta$ is the ray angle, $\omega$ is ray dip, and $\Delta \varphi$ is the difference in the azimuth angles of the ray and the bedding plane. The symmetry axis is along the $x$ axis. The symboll is the ray length and $d$ is the projection of ray element on $x-y$ plane.

\section{Thomsen anisotropy parameter estimation}

An objective function is built from the measured and predicted P-wave ray velocities. This function is minimized using a very fast simulating re-annealing (VFSR) algorithm (Ingber, 1989) in order to estimate Thomsen anisotropy parameters $\varepsilon, \delta$, $\alpha$, and $\beta$,

$$
g=\frac{1}{2}\left(\mathbf{V}-\mathbf{V}^{\prime}\right)\left[\mathbf{C}_{\mathbf{D}}\right]^{-1}\left(\mathbf{V}-\mathbf{V}^{\prime}\right)^{T}
$$

where $g$ is the objective function and $\mathbf{V}$ is the vector of the ray velocities from all the sourcereceiver pairs. $\mathbf{C}_{\mathbf{D}}$ is the covariance matrix of the error in the ray velocities, and $T$ denotes the transpose. The diagonal elements are variances and are the same for all the measurements. Assuming there is no correlation among the residual errors of the ray velocities, the off-diagonal elements (covariance) of the matrix $\mathbf{C}_{\mathbf{D}}$ are zero. Where appropriate, a non-linear conjugate gradient (CG) algorithm is applied after the VFSR to guarantee the convergence to the true solution. Both minimization algorithms require the derivatives of the objective function with respect to the model parameters, namely,

$$
\frac{\partial g}{\partial m_{j}}=-\frac{\partial \mathbf{V}}{\partial m_{j}}\left(\mathbf{V}-\mathbf{V}^{\prime}\right), \quad \frac{d V}{d m}=\left.\frac{d V}{d m}\right|_{p=c o n s .}+\frac{\partial V}{\partial p} \frac{\partial p}{\partial m}, \quad \frac{\partial p}{\partial m}=-\frac{\partial d /\left.\partial m\right|_{p=c o n s .}}{\partial d / \partial p}
$$

For the rays propagating in the isotropy plane, $\partial p / \partial m$ can be directly computed from equation 6 .

\section{Examples}

In the first example, we have computed the noise free P-wave ray velocities for a synthetic VTI model with the Thomsen parameters given in Table 1 ("True" values). We drew a random a priori model from a uniform distribution and ran the VFSR for up to 2000 iterations. We also implemented the CG algorithm on the best estimate obtained from the VFSR. The CG continued for up to 100 iterations. The three purple columns in Table 1 represent the true model and the best estimate from the VFSR and from the CG, respectively. Except $\beta$, the anisotropy parameters are well resolved.

In the second example, laboratory data from a shale specimen cored normal to bedding plane, and subjected to $45 \mathrm{MPa}$ confining pressure and to $5 \mathrm{MPa}$ pore pressure. This time the VFSR was ran for up to 5000 iterations. The uncertainties are computed from the last 1000 candidates. Figures 3 and 4 
show that the convergence of $\varepsilon$ and $\delta$ during the iterations of the VFSR is reasonable, which is likely to provide us with reliable estimates of these anisotropy parameters.

\begin{tabular}{|c|c|c|c|c|c|c|c|}
\hline Parameter & Lower & Upper & True & VFSR & CG & VFSR & $\sigma_{m}$ \\
\hline$\varepsilon$ & 0 & 0.8 & 0.2 & 0.2 & 0.2 & 0.19 & 0.012 \\
\hline$\delta$ & -0.3 & 0.8 & 0.15 & 0.15 & 0.15 & 0.13 & 0.05 \\
\hline$\alpha$ & 1 & 6 & 2.5 & 2.5 & 2.5 & 3.45 & 0.05 \\
\hline$\beta$ & 0.5 & 3 & 1.5 & 1.22 & 1.53 & 1.61 & N/A \\
\hline
\end{tabular}

Table 1 The second and third columns show the hard constraint during the minimization. The columns in purple show the true model, the best estimate from the VFSR, and the estimate from the CG for the synthetic model. The columns in blue show the best estimates and the associated uncertainties from the shale sample. The anisotropy parameters $\alpha$ and $\beta$ are given in $\mathrm{km} / \mathrm{sec}$

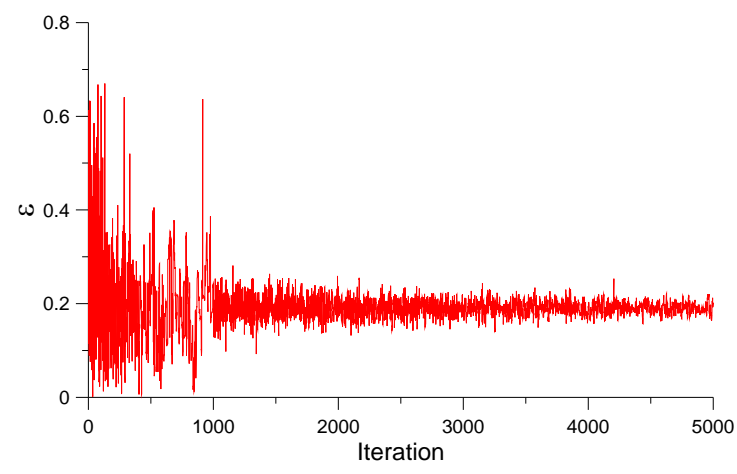

Figure 3 Convergence pattern of the $\varepsilon$ from the VFSR for a shale sample.

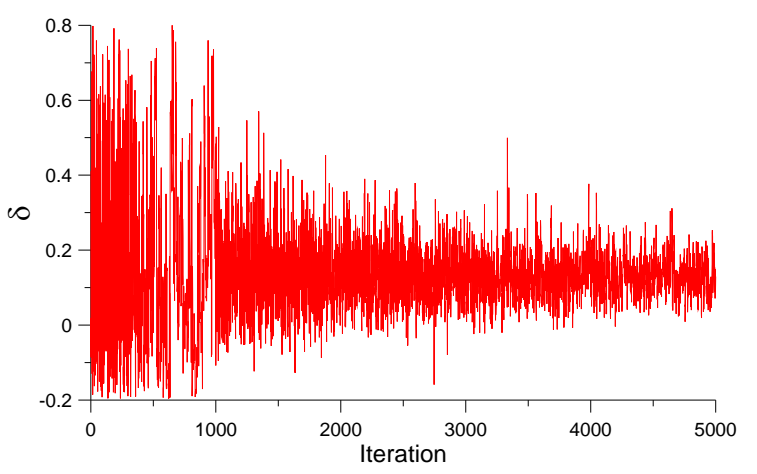

Figure 4 Convergence pattern of the $\delta$ from the VFSR for a shale sample.

\section{Conclusions}

Because of very small sensitivity of the P-wave ray velocities to the S-wave velocity $\beta$, we do not expect to resolve this parameter properly from the VFSR only in real experiments. However, because the P-wave ray velocities from the synthetic model are noise free, the CG was able to converge towards a reasonable estimate of $\beta$. This shows that the objective function in the model space has a unique solution and the anisotropy parameters can be estimated independently. An analysis of the convergence pattern of the anisotropy parameters for the actual shale data, as exemplified for $\varepsilon$ and $\delta$ in Figure 3 and 4, shows that in the presence of noise, $\alpha$ is well estimated, whereas $\varepsilon$ and $\delta$ in particular are estimated with higher uncertainty. This higher uncertainty is attributed to the presence of noise in the experimental data (quality of the picking, heterogeneities within the specimen, etc.). Equation 6 shows that the phase velocity in the isotropy plane is independent of $\delta$ and no measurement along the isotropy plane contributes to the estimation of $\delta$. Hence this estimate is inherently less robust than the estimates of $\varepsilon$ and $\alpha$ because less independent measurements are available. Note that the present methodology, applied here to laboratory scale data, is easily applicable to 3D VSP and surface seismic data at the field scale.

\section{References}

Ingber, L. [1989] Very fast simulated re-annealing. Mathematical and Computer Modelling, 12, 967973.

Thomsen, L. [1986] Weak elastic anisotropy. Geophysics, 51, 1954-1966.

Ursin, B. and Stovas, A. [2006] Traveltime approximations for a layered transversely isotropic medium. Geophysics, 71, D23-D33. 